\title{
Representations by sextenary quadratic forms whose coefficients are 1, 2 and 4
}

by

\author{
Ayşe Alaca, Şaban Alaca, Faruk Uygul \\ and Kenneth S. Williams (Ottawa)
}

1. Introduction. Let $\mathbb{N}, \mathbb{N}_{0}, \mathbb{Z}$ and $\mathbb{C}$ denote the sets of positive integers, nonnegative integers, integers and complex numbers respectively. For $n \in \mathbb{N}_{0}$, and $a_{1}, \ldots, a_{6} \in \mathbb{N}$ we define

$$
N\left(a_{1}, \ldots, a_{6} ; n\right):=\operatorname{card}\left\{\left(x_{1}, \ldots, x_{6}\right) \in \mathbb{Z}^{6} \mid n=a_{1} x_{1}^{2}+\cdots+a_{6} x_{6}^{2}\right\} .
$$

Clearly,

$$
N\left(a_{1}, \ldots, a_{6} ; 0\right)=1 .
$$

As $N\left(a_{1}, \ldots, a_{6} ; n\right)$ is invariant under permutations of $a_{1}, \ldots, a_{6}$, we may suppose that

$$
a_{1} \leq \cdots \leq a_{6} .
$$

There are 21 sextuples $\left(a_{1}, \ldots, a_{6}\right)$ satisfying

$$
a_{1}, \ldots, a_{6} \in\{1,2,4\}, \quad 1=a_{1} \leq a_{2} \leq \cdots \leq a_{6} .
$$

For the 4 sextuples

$$
\left(a_{1}, \ldots, a_{6}\right)=(1,1,1,1,1,1),(1,1,1,1,2,2),(1,1,2,2,2,2),(1,2,2,2,2,4)
$$

it is known that $N\left(a_{1}, \ldots, a_{6} ; n\right)(n \in \mathbb{N})$ can be expressed in terms of the two sums

$$
\begin{aligned}
& G_{4}(n):=\sum_{\substack{d \in \mathbb{N} \\
d \mid n}}\left(\frac{-4}{n / d}\right) d^{2}, \quad n \in \mathbb{N}, \\
& H_{4}(n):=\sum_{\substack{d \in \mathbb{N} \\
d \mid n}}\left(\frac{-4}{d}\right) d^{2}, \quad n \in \mathbb{N},
\end{aligned}
$$

2010 Mathematics Subject Classification: Primary 11E25.

Key words and phrases: sextenary quadratic forms, sum of divisors function, theta functions, infinite products, Gaussian sums. 
where $\left(\frac{-4}{d}\right)(d \in \mathbb{N})$ is the Legendre-Jacobi-Kronecker symbol for discriminant -4 given by

$$
\left(\frac{-4}{d}\right)= \begin{cases}+1 & \text { if } d \equiv 1(\bmod 4) \\ -1 & \text { if } d \equiv 3(\bmod 4) \\ 0 & \text { if } d \equiv 0(\bmod 2)\end{cases}
$$

For $n \in \mathbb{N}$ we have

$$
\begin{aligned}
& N(1,1,1,1,1,1 ; n)=16 G_{4}(n)-4 H_{4}(n), \\
& N(1,1,1,1,2,2 ; n)=8 G_{4}(n)-2\left(1+(-1)^{n}\right) H_{4}(n), \\
& N(1,1,2,2,2,2 ; n)=4 G_{4}(n)-2\left(1+(-1)^{n}\right) H_{4}(n), \\
& N(1,2,2,2,2,4 ; n)=2 G_{4}(n)-4 H_{4}(n / 4)
\end{aligned}
$$

(see [2, Theorem 2.3, p. 551]). (If $f: \mathbb{N} \rightarrow \mathbb{C}$ and $m \notin \mathbb{N}$ we set $f(m)=0$. Thus $H_{4}(n / 4)=0$ if $n \not \equiv 0(\bmod 4)$.) Formula (1.7) is Jacobi's classical formula for the number of representations of a positive integer $n$ as a sum of six squares (see for example [1]).

For the 8 sextuples

$$
\begin{aligned}
\left(a_{1}, \ldots, a_{6}\right)= & (1,1,1,1,1,4),(1,1,1,1,4,4),(1,1,1,2,2,4),(1,1,1,4,4,4), \\
& (1,1,2,2,4,4),(1,1,4,4,4,4),(1,2,2,4,4,4),(1,4,4,4,4,4),
\end{aligned}
$$

$N\left(a_{1}, \ldots, a_{6} ; n\right)(n \in \mathbb{N})$ can be expressed in terms of $G_{4}(n)$ and $H_{4}(n)$ when $n \not \equiv 1(\bmod 4) ;$ however, when $n \equiv 1(\bmod 4)$ the additional sum

$$
I(n):=\sum_{\substack{(x, y) \in \mathbb{Z}^{2} \\ n=x^{2}+4 y^{2}}}\left(x^{2}-4 y^{2}\right), \quad n \in \mathbb{N}, n \equiv 1(\bmod 4),
$$

is required. Let $q \in \mathbb{C}$ be such that $|q|<1$. A basic property of $I(n)$ is

$$
\sum_{\substack{n=1 \\ n \equiv 1(\bmod 4)}}^{\infty} I(n) q^{n}=2 q \prod_{n=1}^{\infty}\left(1-q^{4 n}\right)^{6}
$$

(see [11, Vol. II, p. 377] and [17, p. 122]). We have

$$
\begin{aligned}
& N(1,1,1,1,1,4 ; n)= \begin{cases}6 G_{4}(n)+2 I(n) & \text { if } n \equiv 1(\bmod 4), \\
10 G_{4}(n) & \text { if } n \equiv 2,3(\bmod 4), \\
6 G_{4}(n)-4 H_{4}(n) & \text { if } n \equiv 0(\bmod 4),\end{cases} \\
& N(1,1,1,1,4,4 ; n)= \begin{cases}4 G_{4}(n)+2 I(n) & \text { if } n \equiv 1(\bmod 4), \\
4 G_{4}(n) & \text { if } n \equiv 3(\bmod 4), \\
6 G_{4}(n) & \text { if } n \equiv 2(\bmod 4), \\
2 G_{4}(n)-4 H_{4}(n) & \text { if } n \equiv 0(\bmod 4),\end{cases}
\end{aligned}
$$




$$
\begin{aligned}
& N(1,1,1,4,4,4 ; n)= \begin{cases}3 G_{4}(n)+\frac{3}{2} I(n) & \text { if } n \equiv 1(\bmod 4), \\
G_{4}(n) & \text { if } n \equiv 3(\bmod 4), \\
3 G_{4}(n) & \text { if } n \equiv 2(\bmod 4), \\
G_{4}(n)-4 H_{4}(n) & \text { if } n \equiv 0(\bmod 4),\end{cases} \\
& N(1,1,4,4,4,4 ; n)= \begin{cases}2 G_{4}(n)+I(n) & \text { if } n \equiv 1(\bmod 4), \\
0 & \text { if } n \equiv 3(\bmod 4), \\
G_{4}(n) & \text { if } n \equiv 2(\bmod 4), \\
G_{4}(n)-4 H_{4}(n) & \text { if } n \equiv 0(\bmod 4),\end{cases} \\
& N(1,4,4,4,4,4 ; n)= \begin{cases}G_{4}(n)+\frac{1}{2} I(n) & \text { if } n \equiv 1(\bmod 4), \\
0 & \text { if } n \equiv 2,3(\bmod 4), \\
G_{4}(n)-4 H_{4}(n) & \text { if } n \equiv 0(\bmod 4),\end{cases} \\
& N(1,1,1,2,2,4 ; n)= \begin{cases}4 G_{4}(n)+I(n) & \text { if } n \equiv 1(\bmod 4), \\
4 G_{4}(n) & \text { if } n \equiv 2,3(\bmod 4), \\
4 G_{4}(n)-4 H_{4}(n) & \text { if } n \equiv 0(\bmod 4),\end{cases} \\
& N(1,1,2,2,4,4 ; n)= \begin{cases}2 G_{4}(n)+I(n) & \text { if } n \equiv 1(\bmod 4), \\
2 G_{4}(n) & \text { if } n \equiv 2,3(\bmod 4), \\
2 G_{4}(n)-4 H_{4}(n) & \text { if } n \equiv 0(\bmod 4),\end{cases} \\
& \text { (1.20) } N(1,2,2,4,4,4 ; n)= \begin{cases}G_{4}(n)+\frac{1}{2} I(n) & \text { if } n \equiv 1(\bmod 4), \\
G_{4}(n) & \text { if } n \equiv 2,3(\bmod 4), \\
G_{4}(n)-4 H_{4}(n) & \text { if } n \equiv 0(\bmod 4)\end{cases}
\end{aligned}
$$

(see [3, Theorem 1.1]).

In this paper we consider the remaining $21-4-8=9$ sextuples. For the two sextuples

$$
\left(a_{1}, \ldots, a_{6}\right)=(1,1,1,1,1,2),(1,2,2,2,2,2),
$$

we show that $N\left(a_{1}, \ldots, a_{6} ; n\right)(n \in \mathbb{N})$ can be given in terms of the sums

$$
\begin{array}{ll}
G_{8}(n):=\sum_{\substack{d \in \mathbb{N} \\
d \mid n}}\left(\frac{-8}{n / d}\right) d^{2}, \quad n \in \mathbb{N}, \\
H_{8}(n):=\sum_{\substack{d \in \mathbb{N} \\
d \mid n}}\left(\frac{-8}{d}\right) d^{2}, \quad n \in \mathbb{N},
\end{array}
$$

where $\left(\frac{-8}{d}\right)(d \in \mathbb{N})$ is the Legendre-Jacobi-Kronecker symbol for discriminant -8 , namely

$$
\left(\frac{-8}{d}\right)= \begin{cases}+1 & \text { if } d \equiv 1,3(\bmod 8) \\ -1 & \text { if } d \equiv 5,7(\bmod 8) \\ 0 & \text { if } d \equiv 0(\bmod 2)\end{cases}
$$

In Section 5 we prove the following result. 
TheOREM 1. Let $n \in \mathbb{N}$. Then

$$
\begin{aligned}
& N(1,1,1,1,1,2 ; n)=\frac{32}{3} G_{8}(n)-\frac{2}{3} H_{8}(n), \\
& N(1,2,2,2,2,2 ; n)=\frac{8}{3} G_{8}(n)-\frac{2}{3} H_{8}(n) .
\end{aligned}
$$

The formulas of Theorem 1 were stated but not proved by Liouville [14], [15]. For the remaining $9-2=7$ sextuples, we require for the evaluation of $N\left(a_{1}, \ldots, a_{6} ; n\right)(n \in \mathbb{N})$, in addition to $G_{8}(n)$ and $H_{8}(n)$, the integers $c(n)(n \in \mathbb{N})$ defined by

$$
\sum_{n=1}^{\infty} c(n) q^{n}:=q \prod_{n=1}^{\infty}\left(1-q^{n}\right)^{2}\left(1-q^{2 n}\right)\left(1-q^{4 n}\right)\left(1-q^{8 n}\right)^{2} .
$$

It is known from the work of Martin [16, Table 1, p. 4853] that $c(n)$ is a multiplicative function of $n$. We prove the following result in Section 6 .

Theorem 2. Let $n \in \mathbb{N}$. Then

$$
\begin{aligned}
N(1,1,1,1,2,4 ; n) & \\
& = \begin{cases}\frac{16}{3} G_{8}(n)+\frac{8}{3} c(n) & \text { if } n \equiv 1(\bmod 2), \\
\frac{16}{3} G_{8}(n)-\frac{2}{3} H_{8}(n)-\frac{8}{3} c(n) & \text { if } n \equiv 0(\bmod 2),\end{cases}
\end{aligned}
$$

(ii) $\quad N(1,1,1,2,2,2 ; n)=\frac{16}{3} G_{8}(n)-\frac{2}{3} H_{8}(n)+\frac{4}{3} c(n)$,

(iii) $\quad N(1,1,1,2,4,4 ; n)$

$$
= \begin{cases}\frac{8}{3} G_{8}(n)+\frac{10}{3} c(n) & \text { if } n \equiv 1(\bmod 2), \\ \frac{8}{3} G_{8}(n)-\frac{2}{3} H_{8}(n)-2 c(n) & \text { if } n \equiv 0(\bmod 2),\end{cases}
$$

(iv) $\quad N(1,1,2,2,2,4 ; n)$

$$
= \begin{cases}\frac{8}{3} G_{8}(n)+\frac{4}{3} c(n) & \text { if } n \equiv 1(\bmod 2), \\ \frac{8}{3} G_{8}(n)-\frac{2}{3} H_{8}(n) & \text { if } n \equiv 0(\bmod 2),\end{cases}
$$

(v) $\quad \quad \quad \quad(1,1,2,4,4,4 ; n)$

$$
= \begin{cases}\frac{4}{3} G_{8}(n)+\frac{8}{3} c(n) & \text { if } n \equiv 1(\bmod 2), \\ \frac{4}{3} G_{8}(n)-\frac{2}{3} H_{8}(n)-\frac{2}{3} c(n) & \text { if } n \equiv 0(\bmod 2),\end{cases}
$$

(vi) $\quad N(1,2,2,2,4,4 ; n)$

$$
= \begin{cases}\frac{4}{3} G_{8}(n)+\frac{2}{3} c(n) & \text { if } n \equiv 1(\bmod 2), \\ \frac{4}{3} G_{8}(n)-\frac{2}{3} H_{8}(n)-\frac{2}{3} c(n) & \text { if } n \equiv 0(\bmod 2),\end{cases}
$$

(vii) $\quad N(1,2,4,4,4,4 ; n)$

$$
= \begin{cases}\frac{2}{3} G_{8}(n)+\frac{4}{3} c(n) & \text { if } n \equiv 1(\bmod 2), \\ \frac{2}{3} G_{8}(n)-\frac{2}{3} H_{8}(n) & \text { if } n \equiv 0(\bmod 2) .\end{cases}
$$


Following Ramanujan (see for example [4, p. 6]), we define the theta functions $\varphi(q)$ and $\psi(q)$ by

$$
\varphi(q):=\sum_{n=-\infty}^{\infty} q^{n^{2}}, \quad \psi(q):=\sum_{n=0}^{\infty} q^{n(n+1) / 2} .
$$

The basic properties of $\varphi(q)$ are

$$
\begin{aligned}
\varphi(q)+\varphi(-q) & =2 \varphi\left(q^{4}\right), \\
\varphi^{2}(q)+\varphi^{2}(-q) & =2 \varphi^{2}\left(q^{2}\right), \\
\varphi(q) \varphi(-q) & =\varphi^{2}\left(-q^{2}\right)
\end{aligned}
$$

(see [4, pp. 71, 72, 15]). As simple consequences of Jacobi's triple product identity, we have

$$
\varphi(q)=\prod_{n=1}^{\infty} \frac{\left(1-q^{2 n}\right)^{5}}{\left(1-q^{n}\right)^{2}\left(1-q^{4 n}\right)^{2}}, \quad \varphi(-q)=\prod_{n=1}^{\infty} \frac{\left(1-q^{n}\right)^{2}}{1-q^{2 n}},
$$

and

$$
\psi(q)=\prod_{n=1}^{\infty} \frac{\left(1-q^{2 n}\right)^{2}}{1-q^{n}}
$$

(see for example [10, pp. 282-284]). Setting

$$
E_{k}:=E_{k}(q)=\prod_{n=1}^{\infty}\left(1-q^{k n}\right), \quad k \in \mathbb{N}
$$

we see from (1.29)-(1.31) that

$$
\varphi(q)=\frac{E_{2}^{5}}{E_{1}^{2} E_{4}^{2}}, \quad \varphi(-q)=\frac{E_{1}^{2}}{E_{2}}, \quad \psi(q)=\frac{E_{2}^{2}}{E_{1}}, \quad \varphi\left(q^{2}\right)=\frac{E_{4}^{5}}{E_{2}^{2} E_{8}^{2}},
$$

so that

$$
\varphi(q) \varphi\left(q^{2}\right)=\frac{E_{2}^{3} E_{4}^{3}}{E_{1}^{2} E_{8}^{2}}
$$

From (1.1) and (1.25) we deduce that

$$
\sum_{n=0}^{\infty} N\left(a_{1}, \ldots, a_{6} ; n\right) q^{n}=\varphi\left(q^{a_{1}}\right) \cdots \varphi\left(q^{a_{6}}\right) .
$$

The proofs of Theorems 1 and 2 depend upon the following three results.

Theorem 3. Let $q \in \mathbb{C}$ be such that $|q|<1$. Then

$$
\sum_{n=1}^{\infty} G_{8}(n) q^{n}=\frac{1}{8} \varphi^{5}(q) \varphi\left(q^{2}\right)-\frac{1}{8} \varphi(q) \varphi^{5}\left(q^{2}\right) .
$$


Theorem 4. Let $q \in \mathbb{C}$ be such that $|q|<1$. Then

$$
1-\frac{2}{3} \sum_{n=1}^{\infty} H_{8}(n) q^{n}=-\frac{1}{3} \varphi^{5}(q) \varphi\left(q^{2}\right)+\frac{4}{3} \varphi(q) \varphi^{5}\left(q^{2}\right) .
$$

TheOrem 5. Let $q \in \mathbb{C}$ be such that $|q|<1$. Then

$$
\sum_{n=1}^{\infty} c(n) q^{n}=-\frac{1}{4} \varphi^{5}(q) \varphi\left(q^{2}\right)+\frac{3}{4} \varphi^{3}(q) \varphi^{3}\left(q^{2}\right)-\frac{1}{2} \varphi(q) \varphi^{5}\left(q^{2}\right) .
$$

Theorem 3 is proved in Section 2, Theorem 4 in Section 3 and Theorem 5 in Section 4.

In order to prove Theorems 3 and 4 we need the following results.

Theorem 6. Let $q \in \mathbb{C}$ be such that $|q|<1$. Let $a \in \mathbb{C} \backslash\{0,1,-1\}$ be such that $a \neq q^{n}$ for all $n \in \mathbb{Z}$. Then

$$
\begin{aligned}
& \prod_{n=1}^{\infty} \frac{\left(1-a^{2} q^{n}\right)\left(1-a^{-2} q^{n}\right)\left(1-q^{n}\right)^{6}}{\left(1-a q^{n}\right)^{4}\left(1-a^{-1} q^{n}\right)^{4}} \\
& =1+\frac{(1-a)^{3}}{a(1+a)} \sum_{n=1}^{\infty}\left(\sum_{d \mid n}\left(a^{d}-a^{-d}\right) d^{2}\right) q^{n}
\end{aligned}
$$

Carlitz [5, eq. (1.3), p. 168] derived this theorem from a well-known formula in the theory of elliptic functions for the derivative of the Weierstrass $\wp$-function [20, Question 24, p. 459]. An elementary proof has been given by Dobbie [8].

Theorem 7. Let $a_{1}, a_{2}, a_{3}, b_{1}, b_{2}, b_{3} \in \mathbb{C} \backslash\{0\}$ be such that $a_{i} \neq q^{n} a_{j}$ for all $i, j \in\{1,2,3\}$ with $i \neq j$ and all $n \in \mathbb{Z}$, and $a_{1} a_{2} a_{3}=b_{1} b_{2} b_{3}$. Then

$$
\begin{aligned}
& \frac{\left(1-a_{1} b_{1}^{-1}\right)\left(1-a_{1} b_{2}^{-1}\right)\left(1-a_{1} b_{3}^{-1}\right)}{\left(1-a_{1} a_{2}^{-1}\right)\left(1-a_{1} a_{3}^{-1}\right)} \prod_{j=1}^{\infty}\left(\frac{\left(1-a_{1} b_{1}^{-1} q^{j}\right)\left(1-a_{1}^{-1} b_{1} q^{j}\right)\left(1-a_{1} b_{2}^{-1} q^{j}\right)}{\left(1-a_{1} a_{2}^{-1} q^{j}\right)\left(1-a_{1}^{-1} a_{2} q^{j}\right)}\right. \\
& \left.\times \frac{\left(1-a_{1}^{-1} b_{2} q^{j}\right)\left(1-a_{1} b_{3}^{-1} q^{j}\right)\left(1-a_{1}^{-1} b_{3} q^{j}\right)}{\left(1-a_{1} a_{3}^{-1} q^{j}\right)\left(1-a_{1}^{-1} a_{3} q^{j}\right)}\right)+\frac{\left(1-a_{2} b_{1}^{-1}\right)\left(1-a_{2} b_{2}^{-1}\right)\left(1-a_{2} b_{3}^{-1}\right)}{\left(1-a_{2} a_{1}^{-1}\right)\left(1-a_{2} a_{3}^{-1}\right)} \\
& \times \prod_{j=1}^{\infty} \frac{\left(1-a_{2} b_{1}^{-1} q^{j}\right)\left(1-a_{2}^{-1} b_{1} q^{j}\right)\left(1-a_{2} b_{2}^{-1} q^{j}\right)\left(1-a_{2}^{-1} b_{2} q^{j}\right)\left(1-a_{2} b_{3}^{-1} q^{j}\right)\left(1-a_{2}^{-1} b_{3} q^{j}\right)}{\left(1-a_{2} a_{1}^{-1} q^{j}\right)\left(1-a_{2}^{-1} a_{1} q^{j}\right)\left(1-a_{2} a_{3}^{-1} q^{j}\right)\left(1-a_{2}^{-1} a_{3} q^{j}\right)} \\
& +\frac{\left(1-a_{3} b_{1}^{-1}\right)\left(1-a_{3} b_{2}^{-1}\right)\left(1-a_{3} b_{3}^{-1}\right)}{\left(1-a_{3} a_{1}^{-1}\right)\left(1-a_{3} a_{2}^{-1}\right)} \prod_{j=1}^{\infty}\left(\frac{\left(1-a_{3} b_{1}^{-1} q^{j}\right)\left(1-a_{3}^{-1} b_{1} q^{j}\right)\left(1-a_{3} b_{2}^{-1} q^{j}\right)}{\left(1-a_{3} a_{1}^{-1} q^{j}\right)\left(1-a_{3}^{-1} a_{1} q^{j}\right)}\right. \\
& \left.\times \frac{\left(1-a_{3}^{-1} b_{2} q^{j}\right)\left(1-a_{3} b_{3}^{-1} q^{j}\right)\left(1-a_{3}^{-1} b_{3} q^{j}\right)}{\left(1-a_{3} a_{2}^{-1} q^{j}\right)\left(1-a_{3}^{-1} a_{2} q^{j}\right)}\right)=0 .
\end{aligned}
$$

Theorem 7 is a special case of a result about sigma functions, which was probably known to Weierstrass (see [20, Example 3, p. 451]). It can also be found in or deduced from [18, [19, eq. 7.4.3], [9, Ex. 5.23, p. 138] and [13]. 
Theorem 5 is proved using Berndt's catalogue of theta functions in terms of the parameters $x=1-\varphi^{4}(-q) / \varphi^{4}(q)$ and $z=\varphi^{2}(q)$ (see [4, pp. 122, 123]).

Finally, in Section 7 we show that $c(n)$ defined in (1.24) satisfies the relation $c(2 n)=-2 c(n), n \in \mathbb{N}$.

2. Proof of Theorem 3. Let $q \in \mathbb{C}$ be such that $|q|<1$. For $a, b \in \mathbb{N}$ with $a \leq b$ we define

$$
E_{a, b}=E_{a, b}(q):=\prod_{n=0}^{\infty}\left(1-q^{b n+a}\right) .
$$

From (1.30) and (2.1) we see that

$$
E_{b}=\prod_{n=1}^{\infty}\left(1-q^{b n}\right)=\prod_{n=0}^{\infty}\left(1-q^{b n+b}\right)=E_{b, b} .
$$

If $b \equiv 0(\bmod 2)$, then $b / 2 \in \mathbb{N}, b / 2 \leq b$ and

$$
\begin{aligned}
E_{b} E_{b / 2, b} & =\prod_{n=0}^{\infty}\left(1-q^{b n+b}\right) \prod_{n=0}^{\infty}\left(1-q^{b n+b / 2}\right) \\
& =\prod_{n=0}^{\infty}\left(1-q^{b(2 n+2) / 2}\right)\left(1-q^{b(2 n+1) / 2}\right)=\prod_{n=1}^{\infty}\left(1-q^{b n / 2}\right)
\end{aligned}
$$

so that

$$
E_{b / 2, b}=E_{b / 2} / E_{b}, \quad b \in \mathbb{N}, b \equiv 0(\bmod 2) .
$$

Thus, taking $b=2,4,8$ in (2.3), we obtain

$$
E_{1,2}=E_{1} / E_{2}, \quad E_{2,4}=E_{2} / E_{4}, \quad E_{4,8}=E_{4} / E_{8} .
$$

Let $c \in \mathbb{N}$. We have

$$
b \mathbb{N}_{0}+a=\bigcup_{d=0}^{c-1}\left(b\left(c \mathbb{N}_{0}+d\right)+a\right)=\bigcup_{d=0}^{c-1}\left(b c \mathbb{N}_{0}+(b d+a)\right)
$$

and

$$
\left(b c \mathbb{N}_{0}+(b d+a)\right) \cap\left(b c \mathbb{N}_{0}+\left(b d^{\prime}+a\right)\right)=\emptyset \quad \text { if } 0 \leq d, d^{\prime}<c, d \neq d^{\prime} .
$$

Note that $1 \leq b d+a \leq b c$. Thus (2.1) gives

$$
E_{a, b}=\prod_{d=0}^{c-1} E_{a+b d, b c} .
$$

Taking $a=1, b=2, c=4$ in (2.5), by (2.4) we have

$$
E_{1,8} E_{3,8} E_{5,8} E_{7,8}=E_{1,2}=E_{1} / E_{2} .
$$


Cooper and Hirschhorn ([6, eqs. (1) and (2)] or [7, Theorem 1]) have shown that

$$
\begin{aligned}
\varphi(q)+\varphi\left(q^{2}\right) & =\frac{2 E_{3,8} E_{5,8} E_{8}^{2}}{E_{1,8} E_{7,8} E_{4}}, \\
\varphi(q)-\varphi\left(q^{2}\right) & =2 q \frac{E_{1,8} E_{7,8} E_{8}^{2}}{E_{3,8} E_{5,8} E_{4}} .
\end{aligned}
$$

Multiplying (2.7) and (2.8) together, we obtain

$$
\varphi^{2}(q)-\varphi^{2}\left(q^{2}\right)=4 q \frac{E_{8}^{4}}{E_{4}^{2}} .
$$

From the equations (2.6), (2.7) and (2.8), we have

$$
\begin{aligned}
& \frac{1}{E_{1,8}^{2} E_{7,8}^{2}}=\frac{1}{2}\left(\varphi(q)+\varphi\left(q^{2}\right)\right) \frac{E_{2} E_{4}}{E_{1} E_{8}^{2}}, \\
& \frac{q}{E_{3,8}^{2} E_{5,8}^{2}}=\frac{1}{2}\left(\varphi(q)-\varphi\left(q^{2}\right)\right) \frac{E_{2} E_{4}}{E_{1} E_{8}^{2}} .
\end{aligned}
$$

Thus, appealing to (2.10), (2.11), (1.33) and (2.9), we have

$$
\begin{aligned}
q \frac{E_{2} E_{8}^{6}}{E_{4}}\left(\frac{1}{E_{1,8}^{4} E_{7,8}^{4}}+\right. & \left.\frac{q^{2}}{E_{3,8}^{4} E_{5,8}^{4}}\right) \\
& =\frac{1}{2} q \frac{E_{2}^{3} E_{4} E_{8}^{2}}{E_{1}^{2}}\left(\varphi^{2}(q)+\varphi^{2}\left(q^{2}\right)\right) \\
& =\frac{1}{2} \varphi(q) \varphi\left(q^{2}\right)\left(\varphi^{2}(q)-\varphi^{2}\left(q^{2}\right)\right)\left(\varphi^{2}(q)+\varphi^{2}\left(q^{2}\right)\right)
\end{aligned}
$$

so that

$$
q \frac{E_{2} E_{8}^{6}}{E_{4} E_{1,8}^{4} E_{7,8}^{4}}+q^{3} \frac{E_{2} E_{8}^{6}}{E_{4} E_{3,8}^{4} E_{5,8}^{4}}=\frac{1}{8} \varphi^{5}(q) \varphi\left(q^{2}\right)-\frac{1}{8} \varphi(q) \varphi^{5}\left(q^{2}\right) .
$$

For $a \in\{1,3\}$ we have

$$
\sum_{\substack{e=1 \\ e \equiv a(\bmod 8)}}^{\infty} \sum_{d=1}^{\infty} d^{2} q^{d e}=\sum_{f=0}^{\infty} \sum_{d=1}^{\infty} d^{2} q^{8 d f+a d}=\sum_{d=1}^{\infty} d^{2} q^{a d}+\sum_{f=1}^{\infty} \sum_{d=1}^{\infty} d^{2} q^{8 d f+a d}
$$

that is,

$$
\sum_{\substack{e=1 \\ e \equiv a(\bmod 8)}}^{\infty} \sum_{d=1}^{\infty} d^{2} q^{d e}=\frac{q^{a}\left(1+q^{a}\right)}{\left(1-q^{a}\right)^{3}}+\sum_{n=1}^{\infty}\left(\sum_{d \mid n} d^{2} q^{a d}\right) q^{8 n}
$$

By a similar calculation we have

$$
\sum_{\substack{e=1 \\ e \equiv-a(\bmod 8)}}^{\infty} \sum_{d=1}^{\infty} d^{2} q^{d e}=\sum_{n=1}^{\infty}\left(\sum_{d \mid n} d^{2} q^{-a d}\right) q^{8 n} .
$$


Then, by (1.21), (2.13) and (2.14), we obtain

$$
\begin{aligned}
\sum_{n=1}^{\infty} G_{8}(n) q^{n}= & \sum_{n=1}^{\infty}\left(\sum_{d \mid n}\left(\frac{-8}{n / d}\right) d^{2}\right) q^{n}=\sum_{d, e=1}^{\infty}\left(\frac{-8}{e}\right) d^{2} q^{d e} \\
= & \sum_{a \in\{1,3\}} \sum_{\substack{e=1 \\
e \equiv a(\bmod 8)}}^{\infty} \sum_{d=1}^{\infty} d^{2} q^{d e}-\sum_{a \in\{1,3\}} \sum_{\substack{e=1 \\
e \equiv-a(\bmod 8)}}^{\infty} \sum_{d=1}^{\infty} d^{2} q^{d e} \\
= & \sum_{a \in\{1,3\}}\left(\frac{q^{a}\left(1+q^{a}\right)}{\left(1-q^{a}\right)^{3}}+\sum_{n=1}^{\infty}\left(\sum_{d \mid n} d^{2} q^{a d}\right) q^{8 n}\right) \\
& -\sum_{a \in\{1,3\}} \sum_{n=1}^{\infty}\left(\sum_{d \mid n} d^{2} q^{-a d}\right) q^{8 n},
\end{aligned}
$$

that is,

$$
\begin{aligned}
\sum_{n=1}^{\infty} G_{8}(n) q^{n}= & \sum_{n=1}^{\infty} \sum_{d \mid n} d^{2}\left(q^{d}+q^{3 d}-q^{-d}-q^{-3 d}\right) q^{8 n} \\
& +\frac{q(1+q)}{(1-q)^{3}}+\frac{q^{3}\left(1+q^{3}\right)}{\left(1-q^{3}\right)^{3}} .
\end{aligned}
$$

Replacing $q$ by $q^{8}$ in Carlitz's theorem (Theorem 6) and then taking $a=q$, we obtain

$$
\begin{aligned}
& 1+\frac{(1-q)^{3}}{q(1+q)} \sum_{n=1}^{\infty}\left(\sum_{d \mid n} d^{2}\left(q^{d}-q^{-d}\right)\right) q^{8 n} \\
&=\prod_{n=1}^{\infty} \frac{\left(1-q^{8 n+2}\right)\left(1-q^{8 n-2}\right)\left(1-q^{8 n}\right)^{6}}{\left(1-q^{8 n+1}\right)^{4}\left(1-q^{8 n-1}\right)^{4}}=\frac{(1-q)^{4}}{\left(1-q^{2}\right)} \frac{E_{2,4} E_{8}^{6}}{E_{1,8}^{4} E_{7,8}^{4}},
\end{aligned}
$$

so that by $(2.4)$,

$$
\frac{q(1+q)}{(1-q)^{3}}+\sum_{n=1}^{\infty}\left(\sum_{d \mid n} d^{2}\left(q^{d}-q^{-d}\right)\right) q^{8 n}=q \frac{E_{2} E_{8}^{6}}{E_{4} E_{1,8}^{4} E_{7,8}^{4}} .
$$

Similarly, by taking $a=q^{3}$, we obtain

$$
\frac{q^{3}\left(1+q^{3}\right)}{\left(1-q^{3}\right)^{3}}+\sum_{n=1}^{\infty}\left(\sum_{d \mid n} d^{2}\left(q^{3 d}-q^{-3 d}\right)\right) q^{8 n}=q^{3} \frac{E_{2} E_{8}^{6}}{E_{4} E_{3,8}^{4} E_{5,8}^{4}}
$$

Adding (2.16) and (2.17), and appealing to (2.15), we deduce

$$
\sum_{n=1}^{\infty} G_{8}(n) q^{n}=q \frac{E_{2} E_{8}^{6}}{E_{4} E_{1,8}^{4} E_{7,8}^{4}}+q^{3} \frac{E_{2} E_{8}^{6}}{E_{4} E_{3,8}^{4} E_{5,8}^{4}} .
$$

The required result now follows from (2.12) and (2.18). 
3. Proof of Theorem 4. Let $q \in \mathbb{C}$ be such that $|q|<1$. Substituting the values of $\varphi(q), \varphi(-q)$ and $\varphi\left(q^{2}\right)$ from (1.32) into (1.27), and multiplying by $E_{1}^{4} E_{2}^{4} E_{4}^{4} E_{8}^{4}$, we obtain

$$
E_{2}^{2}\left(E_{2}^{12}+E_{1}^{8} E_{4}^{4}\right) E_{8}^{4}-2 E_{1}^{4} E_{4}^{14}=0 .
$$

Multiplying both sides of this equation by

$$
E_{2}^{2}\left(E_{2}^{12}-E_{1}^{8} E_{4}^{4}\right) E_{8}^{4}+2 E_{1}^{4} E_{4}^{14}
$$

we obtain

$$
E_{2}^{4}\left(E_{2}^{24}-E_{1}^{16} E_{4}^{8}\right) E_{8}^{8}+4 E_{1}^{12} E_{2}^{2} E_{4}^{18} E_{8}^{4}-4 E_{1}^{8} E_{4}^{28}=0 .
$$

Rearranging (3.2) slightly, we have

$$
4 E_{1}^{8} E_{4}^{28}-E_{2}^{28} E_{8}^{8}=4 E_{1}^{12} E_{2}^{2} E_{4}^{18} E_{8}^{4}-E_{1}^{16} E_{2}^{4} E_{4}^{8} E_{8}^{8} .
$$

Dividing both sides of (3.3) by $E_{1}^{16} E_{2}^{4} E_{4}^{10} E_{8}^{6}$, and appealing to (1.32), we deduce

$$
\frac{E_{2}^{4} E_{8}^{2}}{E_{1}^{8} E_{4}^{2}}\left(4 \varphi^{4}\left(q^{2}\right)-\varphi^{4}(q)\right)=4 \frac{E_{4}^{8}}{E_{1}^{4} E_{2}^{2} E_{8}^{2}}-\frac{E_{8}^{2}}{E_{4}^{2}} .
$$

Now let $\omega=e^{2 \pi i / 8}$. We define

$$
\begin{aligned}
& \Pi_{1,7}:=\prod_{n=1}^{\infty}\left(1-\omega q^{n}\right)\left(1-\omega^{7} q^{n}\right), \\
& \Pi_{3,5}:=\prod_{n=1}^{\infty}\left(1-\omega^{3} q^{n}\right)\left(1-\omega^{5} q^{n}\right) .
\end{aligned}
$$

Thus,

$$
\begin{aligned}
\Pi_{1,7} \Pi_{3,5} & =\prod_{n=1}^{\infty}\left(1-\omega q^{n}\right)\left(1-\omega^{3} q^{n}\right)\left(1-\omega^{5} q^{n}\right)\left(1-\omega^{7} q^{n}\right) \\
& =\prod_{n=1}^{\infty}\left(1+q^{4 n}\right)=\prod_{n=1}^{\infty} \frac{\left(1-q^{8 n}\right)}{\left(1-q^{4 n}\right)}
\end{aligned}
$$

so that

$$
\Pi_{1,7} \Pi_{3,5}=E_{8} / E_{4} .
$$

Choosing $a_{1}=1, a_{2}=\omega, a_{3}=\omega^{3}, b_{1}=\omega^{2}, b_{2}=\omega^{4}$ and $b_{3}=\omega^{6}$ in Theorem 7 , we obtain 


$$
\begin{gathered}
\frac{2\left(1+\omega^{2}\right)\left(1-\omega^{2}\right)}{(1+\omega)\left(1+\omega^{3}\right)} \prod_{n=1}^{\infty} \frac{\left(1+q^{n}\right)^{2}\left(1+\omega^{2} q^{n}\right)^{2}\left(1-\omega^{2} q^{n}\right)^{2}}{\left(1-\omega q^{n}\right)\left(1-\omega^{3} q^{n}\right)\left(1-\omega^{5} q^{n}\right)\left(1-\omega^{7} q^{n}\right)} \\
+\frac{(1+\omega)\left(1+\omega^{3}\right)\left(1-\omega^{3}\right)}{(1-\omega)\left(1+\omega^{2}\right)} \prod_{n=1}^{\infty} \frac{\left(1-\omega^{3} q^{n}\right)^{2}\left(1-\omega^{5} q^{n}\right)^{2}}{\left(1+\omega^{2} q^{n}\right)\left(1-\omega^{2} q^{n}\right)} \\
+\frac{(1+\omega)(1-\omega)\left(1+\omega^{3}\right)}{\left(1-\omega^{2}\right)\left(1-\omega^{3}\right)} \prod_{n=1}^{\infty} \frac{\left(1-\omega q^{n}\right)^{2}\left(1-\omega^{7} q^{n}\right)^{2}}{\left(1+\omega^{2} q^{n}\right)\left(1-\omega^{2} q^{n}\right)}=0 .
\end{gathered}
$$

Straightforward calculations show that

$$
\begin{aligned}
\frac{2\left(1+\omega^{2}\right)\left(1-\omega^{2}\right)}{(1+\omega)\left(1+\omega^{3}\right)} & =-2 i \sqrt{2}, \\
\frac{(1+\omega)\left(1+\omega^{3}\right)\left(1-\omega^{3}\right)}{(1-\omega)\left(1+\omega^{2}\right)} & =(\sqrt{2}+1) i, \\
\frac{(1+\omega)(1-\omega)\left(1+\omega^{3}\right)}{\left(1-\omega^{2}\right)\left(1-\omega^{3}\right)} & =(\sqrt{2}-1) i .
\end{aligned}
$$

Appealing to (3.5)-(3.7), we deduce

$$
\begin{gathered}
\prod_{n=1}^{\infty} \frac{\left(1+q^{n}\right)^{2}\left(1+\omega^{2} q^{n}\right)^{2}\left(1-\omega^{2} q^{n}\right)^{2}}{\left(1-\omega q^{n}\right)\left(1-\omega^{3} q^{n}\right)\left(1-\omega^{5} q^{n}\right)\left(1-\omega^{7} q^{n}\right)} \\
=\frac{E_{4}}{E_{8}} \prod_{n=1}^{\infty}\left(1+q^{n}\right)^{2}\left(1+q^{2 n}\right)^{2}=\frac{E_{4}^{3}}{E_{1}^{2} E_{8}}, \\
\prod_{n=1}^{\infty} \frac{\left(1-\omega^{3} q^{n}\right)^{2}\left(1-\omega^{5} q^{n}\right)^{2}}{\left(1+\omega^{2} q^{n}\right)\left(1-\omega^{2} q^{n}\right)}=\Pi_{3,5}^{2} \prod_{n=1}^{\infty} \frac{1}{1+q^{2 n}}=\frac{E_{2}}{E_{4}} \Pi_{3,5}^{2},
\end{gathered}
$$

and

$$
\prod_{n=1}^{\infty} \frac{\left(1-\omega q^{n}\right)^{2}\left(1-\omega^{7} q^{n}\right)^{2}}{\left(1+\omega^{2} q^{n}\right)\left(1-\omega^{2} q^{n}\right)}=\Pi_{1,7}^{2} \prod_{n=1}^{\infty} \frac{1}{1+q^{2 n}}=\frac{E_{2}}{E_{4}} \Pi_{1,7}^{2} .
$$

Thus (3.8) becomes, after dividing by $i E_{2} / E_{4}$,

$$
(\sqrt{2}-1) \Pi_{1,7}^{2}+(\sqrt{2}+1) \Pi_{3,5}^{2}=2 \sqrt{2} \frac{E_{4}^{4}}{E_{1}^{2} E_{2} E_{8}} .
$$

Squaring both sides of (3.9), and appealing to (3.7), we obtain

$$
(3-2 \sqrt{2}) \Pi_{1,7}^{4}+(3+2 \sqrt{2}) \Pi_{3,5}^{4}=\frac{8 E_{4}^{8}}{E_{1}^{4} E_{2}^{2} E_{8}^{2}}-\frac{2 E_{8}^{2}}{E_{4}^{2}} .
$$

Appealing to (3.4), we see that (3.10) becomes

$$
(3-2 \sqrt{2}) \Pi_{1,7}^{4}+(3+2 \sqrt{2}) \Pi_{3,5}^{4}=\frac{2 E_{2}^{4} E_{8}^{2}}{E_{1}^{8} E_{4}^{2}}\left(4 \varphi^{4}\left(q^{2}\right)-\varphi^{4}(q)\right) .
$$


Then, by (3.7), (3.11) and (1.33), we obtain

$$
\begin{aligned}
\frac{(3+2 \sqrt{2})}{6} \frac{E_{1}^{6} E_{4}}{E_{2}} & \Pi_{1,7}^{-4}+\frac{(3-2 \sqrt{2})}{6} \frac{E_{1}^{6} E_{4}}{E_{2}} \Pi_{3,5}^{-4} \\
& =\frac{1}{6} \frac{E_{1}^{6} E_{4}}{E_{2}}\left(\Pi_{1,7} \Pi_{3,5}\right)^{-4}\left((3+2 \sqrt{2}) \Pi_{3,5}^{4}+(3-2 \sqrt{2}) \Pi_{1,7}^{4}\right) \\
& =\frac{1}{6} \frac{E_{1}^{6} E_{4}^{5}}{E_{2} E_{8}^{4}} \frac{2 E_{2}^{4} E_{8}^{2}}{E_{1}^{8} E_{4}^{2}}\left(4 \varphi^{4}\left(q^{2}\right)-\varphi^{4}(q)\right) \\
& =\frac{1}{3} \varphi(q) \varphi\left(q^{2}\right)\left(4 \varphi^{4}\left(q^{2}\right)-\varphi^{4}(q)\right)
\end{aligned}
$$

so that

$$
\begin{aligned}
\frac{(3+2 \sqrt{2})}{6} \frac{E_{1}^{6} E_{4}}{E_{2}} \Pi_{1,7}^{-4}+\frac{(3-2 \sqrt{2})}{6} & \frac{E_{1}^{6} E_{4}}{E_{2}} \Pi_{3,5}^{-4} \\
& =\frac{4}{3} \varphi(q) \varphi^{5}\left(q^{2}\right)-\frac{1}{3} \varphi^{5}(q) \varphi\left(q^{2}\right) .
\end{aligned}
$$

The Gaussian sum for discriminant -8 gives

$$
\omega^{d}+\omega^{3 d}-\omega^{5 d}-\omega^{7 d}=\left(\frac{-8}{d}\right) \sqrt{-8}, \quad d \in \mathbb{N}
$$

(see [12, Theorem 215, p. 221]). Hence

$$
\left(\omega^{d}-\omega^{-d}\right)-\left(\omega^{5 d}-\omega^{-5 d}\right)=\left(\frac{-8}{d}\right) 2 i \sqrt{2}, \quad d \in \mathbb{N} .
$$

Taking $a=\omega$ in Carlitz's theorem (Theorem 6), we obtain after a little rearrangement

$$
\begin{aligned}
\sum_{n=1}^{\infty}\left(\sum_{d \mid n}\left(\omega^{d}-\omega^{-d}\right) d^{2}\right) q^{n} \\
=\frac{\omega(1+\omega)}{(1-\omega)^{3}} \prod_{n=1}^{\infty} \frac{\left(1+q^{2 n}\right)\left(1-q^{n}\right)^{6}}{\left(1-\omega q^{n}\right)^{4}\left(1-\omega^{7} q^{n}\right)^{4}}-\frac{\omega(1+\omega)}{(1-\omega)^{3}}
\end{aligned}
$$

and taking $a=\omega^{5}$ we get

$$
\begin{aligned}
\sum_{n=1}^{\infty}( & \left.\sum_{d \mid n}\left(\omega^{5 d}-\omega^{-5 d}\right) d^{2}\right) q^{n} \\
& =\frac{\omega^{5}\left(1+\omega^{5}\right)}{\left(1-\omega^{5}\right)^{3}} \prod_{n=1}^{\infty} \frac{\left(1+q^{2 n}\right)\left(1-q^{n}\right)^{6}}{\left(1-\omega^{3} q^{n}\right)^{4}\left(1-\omega^{5} q^{n}\right)^{4}}-\frac{\omega^{5}\left(1+\omega^{5}\right)}{\left(1-\omega^{5}\right)^{3}} .
\end{aligned}
$$


Hence, from (3.13)-(3.15), we obtain

$$
\begin{aligned}
& 2 i \sqrt{2} \sum_{n=1}^{\infty} H_{8}(n) q^{n}=2 i \sqrt{2} \sum_{n=1}^{\infty}\left(\sum_{d \mid n}\left(\frac{-8}{d}\right) d^{2}\right) q^{n} \\
&=\sum_{n=1}^{\infty}\left(\sum_{d \mid n}\left(\left(\omega^{d}-\omega^{-d}\right)-\left(\omega^{5 d}-\omega^{-5 d}\right)\right) d^{2}\right) q^{n} \\
&=\frac{\omega(1+\omega)}{(1-\omega)^{3}} \frac{E_{1}^{6} E_{4}}{E_{2}} \Pi_{1,7}^{-4} \\
&-\frac{\omega^{5}\left(1+\omega^{5}\right)}{\left(1-\omega^{5}\right)^{3}} \frac{E_{1}^{6} E_{4}}{E_{2}} \Pi_{3,5}^{-4}-\left(\frac{\omega(1+\omega)}{(1-\omega)^{3}}-\frac{\omega^{5}\left(1+\omega^{5}\right)}{\left(1-\omega^{5}\right)^{3}}\right) \\
&= \frac{-4 i-3 \sqrt{2} i}{2} \frac{E_{1}^{6} E_{4}}{E_{2}} \Pi_{1,7}^{-4}-\frac{-4 i+3 \sqrt{2} i}{2} \frac{E_{1}^{6} E_{4}}{E_{2}} \Pi_{3,5}^{-4}+3 \sqrt{2} i .
\end{aligned}
$$

Dividing both sides by $2 \sqrt{2} i$, we deduce

$$
\sum_{n=1}^{\infty} H_{8}(n) q^{n}=\frac{-3-2 \sqrt{2}}{4} \frac{E_{1}^{6} E_{4}}{E_{2}} \Pi_{1,7}^{-4}+\frac{-3+2 \sqrt{2}}{4} \frac{E_{1}^{6} E_{4}}{E_{2}} \Pi_{3,5}^{-4}+\frac{3}{2} .
$$

Thus, by (3.12), we obtain

$$
\begin{aligned}
1-\frac{2}{3} \sum_{n=1}^{\infty} H_{8}(n) q^{n} & =\frac{3+2 \sqrt{2}}{6} \frac{E_{1}^{6} E_{4}}{E_{2}} \Pi_{1,7}^{-4}+\frac{3-2 \sqrt{2}}{6} \frac{E_{1}^{6} E_{4}}{E_{2}} \Pi_{3,5}^{-4} \\
& =\frac{4}{3} \varphi(q) \varphi^{5}\left(q^{2}\right)-\frac{1}{3} \varphi^{5}(q) \varphi\left(q^{2}\right),
\end{aligned}
$$

as asserted.

4. Proof of Theorem 5. Let

$$
x=1-\frac{\varphi^{4}(-q)}{\varphi^{4}(q)}, \quad z=\varphi^{2}(q) .
$$

From Berndt's catalogue of formulas for theta functions [4, pp. 122, 123], we have

$$
\begin{aligned}
\varphi(q) & =z^{1 / 2}, \\
\varphi(-q) & =(1-x)^{1 / 4} z^{1 / 2}, \\
\varphi\left(q^{2}\right) & =2^{-1 / 2}\left(1+(1-x)^{1 / 2}\right)^{1 / 2} z^{1 / 2}, \\
\psi(q) & =2^{-1 / 2} q^{-1 / 8} x^{1 / 8} z^{1 / 2} .
\end{aligned}
$$

Appealing to (4.2)-(4.5) and (1.32), we obtain 


$$
\begin{aligned}
\frac{E_{2}^{5}}{E_{1}^{2} E_{4}^{2}} & =z^{1 / 2}, \\
\frac{E_{1}^{2}}{E_{2}} & =(1-x)^{1 / 4} z^{1 / 2}, \\
\frac{E_{4}^{5}}{E_{2}^{2} E_{8}^{2}} & =2^{-1 / 2}\left(1+(1-x)^{1 / 2}\right)^{1 / 2} z^{1 / 2}, \\
\frac{E_{2}^{2}}{E_{1}} & =2^{-1 / 2} q^{-1 / 8} x^{1 / 8} z^{1 / 2} .
\end{aligned}
$$

Solving (4.7) and (4.9) for $E_{1}$ and $E_{2}$, then (4.6) for $E_{4}$, and finally (4.8) for $E_{8}$, we obtain

$$
\begin{aligned}
& E_{1}=2^{-1 / 6} q^{-1 / 24} x^{1 / 24}(1-x)^{1 / 6} z^{1 / 2}, \\
& E_{2}=2^{-1 / 3} q^{-1 / 12} x^{1 / 12}(1-x)^{1 / 12} z^{1 / 2}, \\
& E_{4}=2^{-2 / 3} q^{-1 / 6} x^{1 / 6}(1-x)^{1 / 24} z^{1 / 2} \\
& E_{8}=2^{-13 / 12} q^{-1 / 3} x^{1 / 12}(1-x)^{1 / 48}\left(1-(1-x)^{1 / 2}\right)^{1 / 4} z^{1 / 2} .
\end{aligned}
$$

Then, using (4.10)-(4.13), we deduce

$$
\begin{aligned}
E_{2}^{28} E_{8}^{8}+ & 2 E_{1}^{8} E_{4}^{28}+4 q E_{1}^{12} E_{2}^{6} E_{4}^{6} E_{8}^{12}-3 E_{1}^{4} E_{2}^{14} E_{4}^{14} E_{8}^{4} \\
= & 2^{-18} q^{-5} x^{3}(1-x)^{5 / 2}\left(1-(1-x)^{1 / 2}\right)^{2} z^{18}+2^{-19} q^{-5} x^{5}(1-x)^{5 / 2} z^{18} \\
& +2^{-19} q^{-5} x^{3}(1-x)^{3}\left(1-(1-x)^{1 / 2}\right)^{3} z^{18} \\
& \quad-3 \cdot 2^{-19} q^{-5} x^{4}(1-x)^{5 / 2}\left(1-(1-x)^{1 / 2}\right) z^{18} \\
= & 2^{-19} q^{-5} x^{3}(1-x)^{5 / 2} z^{18}\left(2\left(1-(1-x)^{1 / 2}\right)^{2}\right. \\
& \left.+x^{2}+(1-x)^{1 / 2}\left(1-(1-x)^{1 / 2}\right)^{3}-3 x\left(1-(1-x)^{1 / 2}\right)\right)=0 .
\end{aligned}
$$

Thus

$$
q E_{1}^{12} E_{2}^{6} E_{4}^{6} E_{8}^{12}=-\frac{1}{4} E_{2}^{28} E_{8}^{8}+\frac{3}{4} E_{1}^{4} E_{2}^{14} E_{4}^{14} E_{8}^{4}-\frac{1}{2} E_{1}^{8} E_{4}^{28} .
$$

Dividing both sides of (4.14) by $E_{1}^{10} E_{2}^{5} E_{4}^{5} E_{8}^{10}$, we obtain

$$
q E_{1}^{2} E_{2} E_{4} E_{8}^{2}=-\frac{1}{4} \frac{E_{2}^{23}}{E_{1}^{10} E_{4}^{5} E_{8}^{2}}+\frac{3}{4} \frac{E_{2}^{9} E_{4}^{9}}{E_{1}^{6} E_{8}^{6}}-\frac{1}{2} \frac{E_{4}^{23}}{E_{1}^{2} E_{2}^{5} E_{8}^{10}} .
$$

Appealing to (1.24), (1.31), (1.32) and (4.15), we deduce

$$
\sum_{n=1}^{\infty} c(n) q^{n}=-\frac{1}{4} \varphi^{5}(q) \varphi\left(q^{2}\right)+\frac{3}{4} \varphi^{3}(q) \varphi^{3}\left(q^{2}\right)-\frac{1}{2} \varphi(q) \varphi^{5}\left(q^{2}\right)
$$

as required. 
5. Proof of Theorem 1. (i) We have, by Theorems 3 and 4 ,

$$
\begin{aligned}
\sum_{n=0}^{\infty} N( & 1,1,1,1,1,2 ; n) q^{n}=\varphi^{5}(q) \varphi\left(q^{2}\right) \\
& =\left(-\frac{1}{3} \varphi^{5}(q) \varphi\left(q^{2}\right)+\frac{4}{3} \varphi(q) \varphi^{5}\left(q^{2}\right)\right)+\frac{4}{3}\left(\varphi^{5}(q) \varphi\left(q^{2}\right)-\varphi(q) \varphi^{5}\left(q^{2}\right)\right) \\
& =1-\frac{2}{3} \sum_{n=1}^{\infty} H_{8}(n) q^{n}+\frac{32}{3} \sum_{n=1}^{\infty} G_{8}(n) q^{n}
\end{aligned}
$$

so that for $n \in \mathbb{N}$ we get $N(1,1,1,1,1,2 ; n)=\frac{32}{3} G_{8}(n)-\frac{2}{3} H_{8}(n)$, as claimed.

(ii) By Theorems 3 and 4 we have

$$
\begin{aligned}
\sum_{n=0}^{\infty} & N(1,2,2,2,2,2 ; n) q^{n}=\varphi(q) \varphi^{5}\left(q^{2}\right) \\
& =\left(-\frac{1}{3} \varphi^{5}(q) \varphi\left(q^{2}\right)+\frac{4}{3} \varphi(q) \varphi^{5}\left(q^{2}\right)\right)+\left(\frac{1}{3} \varphi^{5}(q) \varphi\left(q^{2}\right)-\frac{1}{3} \varphi(q) \varphi^{5}\left(q^{2}\right)\right) \\
& =1-\frac{2}{3} \sum_{n=1}^{\infty} H_{8}(n) q^{n}+\frac{8}{3} \sum_{n=1}^{\infty} G_{8}(n) q^{n}
\end{aligned}
$$

so that for $n \in \mathbb{N}$ we obtain $N(1,2,2,2,2,2 ; n)=\frac{8}{3} G_{8}(n)-\frac{2}{3} H_{8}(n)$, as asserted.

6. Proof of Theorem 2. In order to prove the formulas of Theorem 2, we introduce the shorthand notation

$$
a:=\varphi(q), \quad b:=\varphi(-q), \quad c:=\varphi\left(q^{2}\right) .
$$

Then, by (1.26), (1.27) and (6.1), we obtain

$$
\varphi\left(q^{4}\right)=\frac{a+b}{2}, \quad c^{2}=\frac{a^{2}+b^{2}}{2} .
$$

By Theorems 3-5, and equations (6.1) and (6.2), we have

$$
\begin{aligned}
\sum_{n=1}^{\infty} G_{8}(n) q^{n} & =\left(\frac{3}{32} a^{5}-\frac{1}{16} a^{3} b^{2}-\frac{1}{32} a b^{4}\right) c, \\
1-\frac{2}{3} \sum_{n=1}^{\infty} H_{8}(n) q^{n} & =\left(\frac{2}{3} a^{3} b^{2}+\frac{1}{3} a b^{4}\right) c, \\
\sum_{n=1}^{\infty} c(n) q^{n} & =\left(\frac{1}{8} a^{3} b^{2}-\frac{1}{8} a b^{4}\right) c .
\end{aligned}
$$


From (6.3)-(6.5) we deduce

$$
\begin{aligned}
a^{5} c & =1+\frac{32}{3} \sum_{n=1}^{\infty} G_{8}(n) q^{n}-\frac{2}{3} \sum_{n=1}^{\infty} H_{8}(n) q^{n}, \\
a^{3} b^{2} c & =1-\frac{2}{3} \sum_{n=1}^{\infty} H_{8}(n) q^{n}+\frac{8}{3} \sum_{n=1}^{\infty} c(n) q^{n}, \\
a b^{4} c & =1-\frac{2}{3} \sum_{n=1}^{\infty} H_{8}(n) q^{n}-\frac{16}{3} \sum_{n=1}^{\infty} c(n) q^{n} .
\end{aligned}
$$

Under the mapping $q \mapsto-q$, we have $a \mapsto b, b \mapsto a$ and $c \mapsto c$. Thus (6.6)-(6.8) yield

$$
\begin{aligned}
a^{4} b c & =1-\frac{2}{3} \sum_{n=1}^{\infty} H_{8}(n)(-q)^{n}-\frac{16}{3} \sum_{n=1}^{\infty} c(n)(-q)^{n}, \\
a^{2} b^{3} c & =1-\frac{2}{3} \sum_{n=1}^{\infty} H_{8}(n)(-q)^{n}+\frac{8}{3} \sum_{n=1}^{\infty} c(n)(-q)^{n}, \\
b^{5} c & =1+\frac{32}{3} \sum_{n=1}^{\infty} G_{8}(n)(-q)^{n}-\frac{2}{3} \sum_{n=1}^{\infty} H_{8}(n)(-q)^{n} .
\end{aligned}
$$

(i) Evaluation of $N(1,1,1,1,2,4 ; n)$. Appealing to (1.34), (6.1), (6.6) and (6.9), we obtain

$$
\begin{aligned}
\sum_{n=0}^{\infty} N(1,1,1,1,2,4 ; n) q^{n} \\
=\varphi^{4}(q) \varphi\left(q^{2}\right) \varphi\left(q^{4}\right)=\frac{1}{2} a^{5} c+\frac{1}{2} a^{4} b c \\
=1+\sum_{n=1}^{\infty}\left(\frac{16}{3} G_{8}(n)-\frac{1}{3}\left(1+(-1)^{n}\right) H_{8}(n)-\frac{8}{3}(-1)^{n} c(n)\right) q^{n}
\end{aligned}
$$

so that, for $n \in \mathbb{N}$,

$$
\begin{aligned}
N(1,1,1,1,2,4 ; n) & =\frac{16}{3} G_{8}(n)-\frac{1}{3}\left(1+(-1)^{n}\right) H_{8}(n)-\frac{8}{3}(-1)^{n} c(n) \\
& = \begin{cases}\frac{16}{3} G_{8}(n)+\frac{8}{3} c(n) & \text { if } n \equiv 1(\bmod 2), \\
\frac{16}{3} G_{8}(n)-\frac{2}{3} H_{8}(n)-\frac{8}{3} c(n) & \text { if } n \equiv 0(\bmod 2),\end{cases}
\end{aligned}
$$

as asserted.

(ii) Evaluation of $N(1,1,1,2,2,2 ; n)$. Appealing to (1.34), (6.1), (6.2), (6.6) and (6.7), we deduce that

$$
\begin{aligned}
\sum_{n=0}^{\infty} N(1,1,1,2,2,2 ; n) q^{n} & =\varphi^{3}(q) \varphi^{3}\left(q^{2}\right)=\frac{1}{2} a^{5} c+\frac{1}{2} a^{3} b^{2} c \\
& =1+\sum_{n=1}^{\infty}\left(\frac{16}{3} G_{8}(n)-\frac{2}{3} H_{8}(n)+\frac{4}{3} c(n)\right) q^{n}
\end{aligned}
$$


so that $N(1,1,1,2,2,2 ; n)=\frac{16}{3} G_{8}(n)-\frac{2}{3} H_{8}(n)+\frac{4}{3} c(n)$ for $n \in \mathbb{N}$, as asserted.

(iii) Evaluation of $N(1,1,1,2,4,4 ; n)$. Appealing to (1.34), (6.1), (6.2), (6.6), (6.7) and (6.9), we obtain

$$
\begin{aligned}
\sum_{n=0}^{\infty} N(1,1, & 1,2,4,4 ; n) q^{n} \\
& =\varphi^{3}(q) \varphi\left(q^{2}\right) \varphi^{2}\left(q^{4}\right)=\frac{1}{4} a^{5} c+\frac{1}{2} a^{4} b c+\frac{1}{4} a^{3} b^{2} c \\
& =1+\sum_{n=1}^{\infty}\left(\frac{8}{3} G_{8}(n)-\frac{1}{3}\left(1+(-1)^{n}\right) H_{8}(n)+\frac{2}{3}\left(1-4(-1)^{n}\right) c(n)\right) q^{n}
\end{aligned}
$$

so that, for $n \in \mathbb{N}$,

$$
\begin{aligned}
N(1,1,1,2,4,4 ; n) & =\frac{8}{3} G_{8}(n)-\frac{1}{3}\left(1+(-1)^{n}\right) H_{8}(n)+\frac{2}{3}\left(1-4(-1)^{n}\right) c(n) \\
& = \begin{cases}\frac{8}{3} G_{8}(n)+\frac{10}{3} c(n) & \text { if } n \equiv 1(\bmod 2), \\
\frac{8}{3} G_{8}(n)-\frac{2}{3} H_{8}(n)-2 c(n) & \text { if } n \equiv 0(\bmod 2),\end{cases}
\end{aligned}
$$

as asserted.

(iv) Evaluation of $N(1,1,2,2,2,4 ; n)$. Appealing to (1.34), (6.1), (6.2), (6.6), (6.7), (6.9) and (6.10), we obtain

$$
\begin{aligned}
\sum_{n=0}^{\infty} N(1,1,2,2,2,4 ; n) q^{n} \\
=\varphi^{2}(q) \varphi^{3}\left(q^{2}\right) \varphi\left(q^{4}\right)=\frac{1}{4} a^{5} c+\frac{1}{4} a^{4} b c+\frac{1}{4} a^{3} b^{2} c+\frac{1}{4} a^{2} b^{3} c \\
=1+\sum_{n=1}^{\infty}\left(\frac{8}{3} G_{8}(n)-\frac{1}{3}\left(1+(-1)^{n}\right) H_{8}(n)+\frac{2}{3}\left(1-(-1)^{n}\right) c(n)\right) q^{n}
\end{aligned}
$$

so that, for $n \in \mathbb{N}$,

$$
\begin{aligned}
N(1,1,2,2,2,4 ; n) & =\frac{8}{3} G_{8}(n)-\frac{1}{3}\left(1+(-1)^{n}\right) H_{8}(n)+\frac{2}{3}\left(1-(-1)^{n}\right) c(n) \\
& = \begin{cases}\frac{8}{3} G_{8}(n)+\frac{4}{3} c(n) & \text { if } n \equiv 1(\bmod 2), \\
\frac{8}{3} G_{8}(n)-\frac{2}{3} H_{8}(n) & \text { if } n \equiv 0(\bmod 2),\end{cases}
\end{aligned}
$$

as asserted.

(v) Evaluation of $N(1,1,2,4,4,4 ; n)$. Appealing to (1.34), (6.1), (6.2), (6.6), (6.7), (6.9) and (6.10), we obtain

$$
\begin{aligned}
\sum_{n=0}^{\infty} N(1,1, & 2,4,4,4 ; n) q^{n} \\
& =\varphi^{2}(q) \varphi\left(q^{2}\right) \varphi^{3}\left(q^{4}\right)=\frac{1}{8} a^{5} c+\frac{3}{8} a^{4} b c+\frac{3}{8} a^{3} b^{2} c+\frac{1}{8} a^{2} b^{3} c \\
& =1+\sum_{n=1}^{\infty}\left(\frac{4}{3} G_{8}(n)-\frac{1}{3}\left(1+(-1)^{n}\right) H_{8}(n)+\frac{1}{3}\left(3-5(-1)^{n}\right) c(n)\right) q^{n}
\end{aligned}
$$


so that, for $n \in \mathbb{N}$,

$$
\begin{aligned}
N(1,1,2,4,4,4 ; n) & =\frac{4}{3} G_{8}(n)-\frac{1}{3}\left(1+(-1)^{n}\right) H_{8}(n)+\frac{1}{3}\left(3-5(-1)^{n}\right) c(n) \\
& = \begin{cases}\frac{4}{3} G_{8}(n)+\frac{8}{3} c(n) & \text { if } n \equiv 1(\bmod 2), \\
\frac{4}{3} G_{8}(n)-\frac{2}{3} H_{8}(n)-\frac{2}{3} c(n) & \text { if } n \equiv 0(\bmod 2),\end{cases}
\end{aligned}
$$

as asserted.

(vi) Evaluation of $N(1,2,2,2,4,4 ; n)$. Appealing to (1.34), (6.1), (6.2) and (6.6)-(6.10), we obtain

$$
\begin{aligned}
\sum_{n=0}^{\infty} N(1,2,2,2,4,4 ; n) q^{n} & \\
& =\varphi(q) \varphi^{3}\left(q^{2}\right) \varphi^{2}\left(q^{4}\right)=\frac{1}{8} a^{5} c+\frac{1}{4} a^{4} b c+\frac{1}{4} a^{3} b^{2} c+\frac{1}{4} a^{2} b^{3} c+\frac{1}{8} a b^{4} c \\
& =1+\sum_{n=1}^{\infty}\left(\frac{4}{3} G_{8}(n)-\frac{1}{3}\left(1+(-1)^{n}\right) H_{8}(n)-\frac{2}{3}(-1)^{n} c(n)\right) q^{n}
\end{aligned}
$$

so that, for $n \in \mathbb{N}$,

$$
\begin{aligned}
N(1,2,2,2,4,4 ; n) & =\frac{4}{3} G_{8}(n)-\frac{1}{3}\left(1+(-1)^{n}\right) H_{8}(n)-\frac{2}{3}(-1)^{n} c(n) \\
& = \begin{cases}\frac{4}{3} G_{8}(n)+\frac{2}{3} c(n) & \text { if } n \equiv 1(\bmod 2), \\
\frac{4}{3} G_{8}(n)-\frac{2}{3} H_{8}(n)-\frac{2}{3} c(n) & \text { if } n \equiv 0(\bmod 2),\end{cases}
\end{aligned}
$$

as asserted.

(vii) Evaluation of $N(1,2,4,4,4,4 ; n)$. Appealing to (1.34), (6.1), (6.2) and (6.6)-(6.10), we obtain

$$
\begin{aligned}
\sum_{n=0}^{\infty} N(1,2, & 4,4,4,4 ; n) q^{n} \\
& =\varphi(q) \varphi\left(q^{2}\right) \varphi^{4}\left(q^{4}\right)=\frac{1}{16} a^{5} c+\frac{1}{4} a^{4} b c+\frac{3}{8} a^{3} b^{2} c+\frac{1}{4} a^{2} b^{3} c+\frac{1}{16} a b^{4} c \\
& =1+\sum_{n=1}^{\infty}\left(\frac{2}{3} G_{8}(n)-\frac{1}{3}\left(1+(-1)^{n}\right) H_{8}(n)+\frac{2}{3}\left(1-(-1)^{n}\right) c(n)\right) q^{n}
\end{aligned}
$$

so that, for $n \in \mathbb{N}$,

$$
\begin{aligned}
N(1,2,4,4,4,4 ; n) & =\frac{2}{3} G_{8}(n)-\frac{1}{3}\left(1+(-1)^{n}\right) H_{8}(n)+\frac{2}{3}\left(1-(-1)^{n}\right) c(n) \\
& = \begin{cases}\frac{2}{3} G_{8}(n)+\frac{4}{3} c(n) & \text { if } n \equiv 1(\bmod 2), \\
\frac{2}{3} G_{8}(n)-\frac{2}{3} H_{8}(n) & \text { if } n \equiv 0(\bmod 2),\end{cases}
\end{aligned}
$$

as asserted. 
7. A property of $c(n)$. We close by proving the following property of $c(n)$ :

$$
c(2 n)=-2 c(n), \quad n \in \mathbb{N} .
$$

As a consequence of (7.1) we have (as $c(1)=1$ )

$$
c\left(2^{k}\right)=(-1)^{k} 2^{k}, \quad k \in \mathbb{N}_{0} .
$$

Proof of (7.1). From (1.31) we have

$$
\begin{aligned}
E_{1}(-q) & =\prod_{n=1}^{\infty}\left(1-(-q)^{n}\right)=\prod_{n=1}^{\infty}\left(1-q^{2 n}\right)\left(1+q^{2 n-1}\right) \\
& =E_{2} \prod_{n=1}^{\infty} \frac{1+q^{n}}{1+q^{2 n}}=E_{2} \prod_{n=1}^{\infty} \frac{1-q^{2 n}}{1-q^{n}} \frac{1-q^{2 n}}{1-q^{4 n}}
\end{aligned}
$$

so that

$$
E_{1}(-q)=\frac{E_{2}^{3}}{E_{1} E_{4}} .
$$

Now, by (1.24) and (1.31), we have

$$
\sum_{n=1}^{\infty} c(n) q^{n}=q E_{1}^{2} E_{2} E_{4} E_{8}^{2}
$$

so that, by (7.3),

$$
\sum_{n=1}^{\infty} c(n)(-q)^{n}=-q \frac{E_{2}^{7} E_{8}^{2}}{E_{1}^{2} E_{4}} .
$$

Thus, by (7.4) and (7.5), we obtain

$$
\begin{aligned}
\sum_{n=1}^{\infty} c(2 n) q^{2 n} & =\frac{1}{2} \sum_{n=1}^{\infty} c(n) q^{n}+\frac{1}{2} \sum_{n=1}^{\infty} c(n)(-q)^{n} \\
& =-\frac{1}{2} q E_{2}^{2} E_{4} E_{8}^{2}\left(\frac{E_{2}^{5}}{E_{1}^{2} E_{4}^{2}}-\frac{E_{1}^{2}}{E_{2}}\right) .
\end{aligned}
$$

Appealing to (1.32), we deduce that

$$
\sum_{n=1}^{\infty} c(2 n) q^{2 n}=-\frac{1}{2} q E_{2}^{2} E_{4} E_{8}^{2}(\varphi(q)-\varphi(-q)) .
$$

Recalling that

$$
\varphi(q)-\varphi(-q)=4 q \psi\left(q^{8}\right)
$$

(see for example [4, p. 71]) and that

$$
\psi\left(q^{8}\right)=\frac{E_{16}^{2}}{E_{8}}
$$


from (1.32) with $q$ replaced by $q^{8}$, we obtain (by (7.4))

$$
\sum_{n=1}^{\infty} c(2 n) q^{2 n}=-2 q^{2} E_{2}^{2} E_{4} E_{8} E_{16}^{2}=-2 \sum_{n=1}^{\infty} c(n) q^{2 n},
$$

from which we deduce (7.1) on equating coefficients of $q^{2 n}(n \in \mathbb{N})$.

Numerical evidence suggests that for an odd prime $p$ and $n \in \mathbb{N}$, we have

$$
c\left(p^{n}\right)=c(p) c\left(p^{n-1}\right)-p^{2} c\left(p^{n-2}\right) \quad \text { if }\left(\frac{-8}{p}\right)=1 \text { and } n \geq 2,
$$

and

$$
c\left(p^{n}\right)=\frac{1}{2}\left(1+(-1)^{n}\right) p^{n} \quad \text { if }\left(\frac{-8}{p}\right)=-1 .
$$

Acknowledgements. The research of the second and fourth authors was supported by research grants from the Natural Sciences and Engineering Research Council of Canada.

\section{References}

[1] A. Alaca, S. Alaca and K. S. Williams, The simplest proof of Jacobi's six squares theorem, Far East J. Math. Sci. 27 (2007), 187-192.

[2] - - - - Liouville's sextenary quadratic forms $x^{2}+y^{2}+z^{2}+t^{2}+2 u^{2}+2 v^{2}$, $x^{2}+y^{2}+2 z^{2}+2 t^{2}+2 u^{2}+2 v^{2}$ and $x^{2}+2 y^{2}+2 z^{2}+2 t^{2}+2 u^{2}+4 v^{2}$, ibid. 30 (2008), $547-556$.

[3] - , 一, 一, Sextenary quadratic forms and an identity of Klein and Fricke, Int. J. Number Theory, to appear.

[4] B. C. Berndt, Number Theory in the Spirit of Ramanujan, Amer. Math. Soc., Providence, RI, 2006.

[5] L. Carlitz, Note on some partition formulae, Quart. J. Math. Oxford Ser. (2) 4 (1953), 168-172.

[6] S. Cooper and M. Hirschhorn, On some infinite product identities, Rocky Mountain J. Math. 31 (2001), 131-139.

[7] - - - On some sum-to-product identities, Bull. Austral. Math. Soc. 63 (2001), 353-365.

[8] J. M. Dobbie, A simple proof of some partition formulae of Ramanujan's, Quart. J. Math. Oxford Ser. (2) 6 (1955), 193-196.

[9] G. Gasper and M. Rahman, Basic Hypergeometric Series, Cambridge Univ. Press, 1990.

[10] G. H. Hardy and E. M. Wright, An Introduction to the Theory of Numbers, 4th ed., Oxford Univ. Press, Oxford, 1960.

[11] F. Klein und R. Fricke, Vorlesungen über die Theorie der elliptischen Modulfunctionen, Vols. I, II, Teubner, Leipzig, 1890, 1892.

[12] E. Landau, Elementary Number Theory, Chelsea, New York, 1958.

[13] R. Lewis, On the ranks of partitions modulo 9, Bull. London Math. Soc. 23 (1991), 417-421.

[14] J. Liouville, Sur la forme $x^{2}+y^{2}+z^{2}+t^{2}+u^{2}+2 v^{2}$, J. Math. Pures Appl. 9 (1864), 161-174. 
[15] J. Liouville, Sur la forme $x^{2}+2\left(y^{2}+z^{2}+t^{2}+u^{2}+v^{2}\right)$, ibid. 9 (1864), 175-180.

[16] Y. Martin, Multiplicative $\eta$-quotients, Trans. Amer. Math. Soc. 348 (1996), 48254856.

[17] L. J. Mordell, On Mr. Ramanujan's empirical expansions of modular functions, Proc. Cambridge Philos. Soc. 19 (1917), 117-124.

[18] L. Slater, A note on equivalent product theorems, Math. Gazette 38 (1954), 127-128.

[19] -, Generalized Hypergeometric Functions, Cambridge Univ. Press, Cambridge, 1966.

[20] E. T. Whittaker and G. N. Watson, A Course of Modern Analysis, 4th ed., Cambridge Univ. Press, Cambridge, 1962.

Ayşe Alaca, Şaban Alaca, Faruk Uygul, Kenneth S. Williams

Centre for Research in Algebra and Number Theory

School of Mathematics and Statistics

Carleton University

Ottawa, Ontario, Canada K1S 5B6

E-mail: aalaca@connect.carleton.ca

salaca@connect.carleton.ca

fuygul@connect.carleton.ca

kwilliam@connect.carleton.ca

Received on 3.4.2009

and in revised form on 16.5.2009 